\title{
Structure of Dense Liquid Water by Neutron Scattering to 6.5 GPa and $670 \mathrm{~K}$
}

\author{
Th. Strässle, ${ }^{1, *}$ A. M. Saitta, ${ }^{1}$ Y. Le Godec, ${ }^{1}$ G. Hamel, ${ }^{1}$ S. Klotz, ${ }^{1}$ J. S. Loveday, ${ }^{2}$ and R. J. Nelmes ${ }^{2}$ \\ ${ }^{1}$ Physique des Milieux Denses, IMPMC, CNRS-UMR 7590, Université Pierre et Marie Curie, 75252 Paris, France \\ ${ }^{2}$ SUPA, School of Physics and Centre for Science at Extreme Conditions, The University of Edinburgh, \\ Edinburgh EH9 3JZ, United Kingdom
}

(Received 16 July 2005; published 13 February 2006)

\begin{abstract}
We present a neutron diffraction study of liquid water to $6.5 \mathrm{GPa}$ and $670 \mathrm{~K}$. From the measured structure factors we determine radial and angular distributions. It is shown that with increasing density water approaches a local structure common to a simple liquid while distorting only a little the tetrahedral first-neighbor coordination imposed by hydrogen bonds that remain intact.
\end{abstract}

The hydrogen bonding in water favors a tetrahedral arrangement of nearest water molecules which results in a three-dimensional random hydrogen-bonded network characterized by a relatively open structure. This differs substantially from structures adopted by liquids with simpler interactions. It was suggested that the discrepancy between open-network forming and more compact local structures may be at the very origin of the unusual thermodynamic behavior of liquid water such as, e.g., its density maximum, compressibility minimum, and specific heat minimum [[1] and references therein]. While these experimental observations were taken as evidence for a second critical point in water [2], other models [3-5] have been proposed which equally may explain the aforementioned anomalies. Support for models based on two states of water has indeed been given by neutron diffraction (ND) [6] and Raman [7] studies under pressure as well as by classical molecular dynamics (MD) calculations [8]. Although different conclusions are drawn from these studies, they reveal the possible existence of a high-density state of liquid water (HDW) which at a pressure of a few kilobars gradually evolves at the expense of a low-density state (LDW). The highest pressure water has been studied so far by diffraction methods is $1.6 \mathrm{GPa}$ [9]. This ND study revealed an increase of the coordination number with increasing pressure as the second neighbor molecules approached a central molecule while first-neighbor molecules distances were essentially unaffected. This and more recent studies to lower pressures associated the local structure of liquid water with those of the ice phases just below the melting line [10], as well as those of the amorphous ice phases (LDA and HDA) [11]. All diffraction studies on water reveal a distinct change in $S(Q)$ already within the first few kilobars, which has been interpreted as a collapse of the second neighbor shell towards the firstneighbor shell and which would involve a breaking of the hydrogen bond ( $\mathrm{H}$ bond) between these two shells [6]. An apparent decrease or weakening of the $\mathrm{H}$ bond with increasing pressure (or temperature) has been indicated by various indirect means, e.g., decrease of the $\sim 4.5 \AA$ peak in the $g(r)$ of $\mathrm{x}$-ray data $[10,12]$, the ratio of the high- frequency and hydrodynamic limit of the sound velocity [13], x-ray absorption spectroscopy, and x-ray Raman scattering [14]. However, these findings partly contradict results from $a b$ initio MD calculations [15] and ND [11].

Here we report detailed neutron diffraction results on liquid water to $6.5 \mathrm{GPa}$-much extending the previous limit of $1.6 \mathrm{GPa}$ [9]. Our results give a clear picture of the structural evolution of water under extreme conditions which can be directly compared to predictions from numerical methods. To prevent pressure induced freezing, measurements were carried out at pressure/temperature $(P / T)$ points at approximately constant distance above the melting line (inset Fig. 1). We find that at high- $P / T$ similarities to structures known from simple liquids can be identified although the $\mathrm{H}$ bonds remain intact. We use the term intact $H$ bond in the sense that signatures in the local structure of water may be traced back to having their very origin in the peculiarities of the $\mathrm{H}$ bond.

Structure factors $S(Q)$ of $\mathrm{D}_{2} \mathrm{O}$ were measured on the PEARL/HiPr TOF diffractometer at the ISIS neutron source, UK. A dedicated high- $P / T$ setup of the ParisEdinburgh pressure cell [described in detail elsewhere [16]] allowed the internal heating of $\sim 45 \mathrm{~mm}^{3} \mathrm{D}_{2} \mathrm{O}$ $(99.96 \%)$ up to $670 \mathrm{~K}$ at $6.5 \mathrm{GPa}$. Pressures were determined to $\pm 0.2 \mathrm{GPa}$ by the known equation of state of the $\mathrm{MgO}$ pressure calibrant. All temperatures were measured to $\pm 20 \mathrm{~K}$ by in situ neutron absorption resonance from $\mathrm{Ta}$ in contact with the sample container. Data were corrected for background and wavelength-dependent attenuation of the anvil-gasket assembly material through measurements in strictly the same gasket-anvil-cell geometry, first with no sample and then with the sample replaced by a cylinder of vanadium. Figure 1 shows three normalized $S(Q)$ representative for the total of 7 measured $P / T$ points. We note that the $S(Q)$ at ambient conditions is in excellent agreement with earlier data $[6,11]$ despite the limited sample size of our high- $P / T$ setup. The measured $S(Q)$ were then analyzed by the empirical potential structural refinement (EPSR) method [12], similar to previous measurements on water [6] and amorphous ice [17] under pressure. EPSR is based on a MD technique using a standard reference po- 
tential together with an additional superimposed empirical potential. The latter is refined in order to fit the experimentally determined $S(Q)$ (Fig. 1). We used the SPC/E reference potential on a box of 2000 molecules and at number densities determined from the Saul-Wagner equation of state of water [18]. The resulting box of molecules was then analyzed in terms of radial and angular distributions. Importantly, EPSR allows us to determine also partial distribution functions from the measured $\mathrm{D}_{2} \mathrm{O} S(Q)$. This information could be obtained more directly from measurements of all three isotope substituted samples, $\mathrm{D}_{2} \mathrm{O}$, $\mathrm{H}_{2} \mathrm{O}$, and HDO, but there is no practical way that such measurements could be carried out with sufficient precision under the extreme conditions of this study. The only feasible way to do it is thus to use the structural modeling, and take care to check it against results from other techniques. In this case, we compared with results from isotope substituted studies at ambient pressure and computational modeling at high pressure.

Figure 2 shows the three intermolecular partial radial distribution functions $g_{\alpha \beta}(r)$ and the coordination numbers $n_{\mathrm{OO}}(r)$ and $n_{\mathrm{OD}}(r)$. The $g_{\mathrm{OO}}$ at ambient conditions may be directly compared to earlier neutron [12] and x-ray studies [19], and recent $a b$ initio calculations [20,21], as depicted in the upper inset of Fig. 2. We find good agreement with our ambient pressure data, including Hura et al. 's x ray $g_{\mathrm{OO}}$ [20], which was determined by standard Fourier transform methods. From this we draw the important conclusion that our EPSR analysis is reliable, even for the determination of the oxygen-oxygen correlations, which for neutrons contribute only $9 \%$ to the overall signal. At elevated $P / T$, a

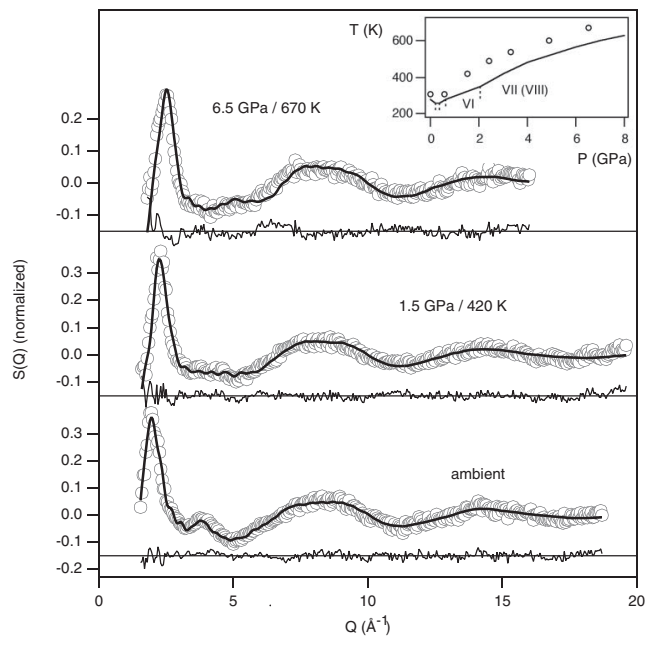

FIG. 1. Measured $S(Q)$ of $\mathrm{D}_{2} \mathrm{O}$ together with EPSR fits (solid lines) and corresponding residua for three representative data sets. The acquisition time was $\sim 18 \mathrm{~h}$ for each data set. The inset shows the phase diagram with $P / T$ conditions where data were taken. The $Q$ range below $1.5 \AA^{-1}$ is inaccessible at the experimental scattering angle of $90^{\circ}$ [16]; variations in the upper limit arise from small differences in the experimental setups under the different $P / T$ conditions. pronounced broadening of the first peak in $g_{\mathrm{OO}}$, a decrease of the first peak and a broadening of the second peak in $g_{\text {OD }}$, and an overall decrease of the modulation amplitude of $g_{\mathrm{DD}}$ are observed. The oxygen-oxygen coordination number $n_{\mathrm{OO}}$ at the second inflection point $(\sim 3.2 \AA$ at ambient and $\sim 3.8 \AA$ at $6.5 \mathrm{GPa} / 670 \mathrm{~K}$ ) changes from a value slightly above 4 at ambient to a value close to 12 at the highest investigated $P / T$. These findings show the same tendency as found in earlier ab initio calculations carried out up to similar densities at constant $T=600 \mathrm{~K}$ [15]. Figure 2 contains two remarkable and unexpected features in the $g_{\mathrm{OO}}$ and $n_{\mathrm{OD}}$ : First, there is a striking similarity of the $g_{\mathrm{OO}}$ at high $P / T$ to that of a simple monoatomic liquid, as shown in Fig. 3 where the $g_{\mathrm{OO}}(r)$ of water are compared with the $g(r)$ of liquid argon [22]. The agreement is remarkable, given that the comparison between the data is completely parameter-free. And second, the $n_{\mathrm{OD}}(r)$ coordination number up to $2.2 \AA$ (see arrow Fig. 2) is almost density independent, suggesting the existence of $\mathrm{H}$ bonds up to the highest pressures. These two findings seem to suggest that water at high densities approaches a structure common to simple liquids while maintaining its $\mathrm{H}$ bonds, two features which appear at first glance to be incompatible. We emphasize at this point that when data at different density $\rho$ are compared, a mere decrease of the height of the first peak in $g_{\mathrm{OD}}$ is not indicative of a decrease of $\mathrm{H}$ bonds. In fact, the number of $\mathrm{H}$ bonds is rather reflected by $n_{\mathrm{OD}}(r)=4 \pi \rho \int_{0}^{r} r^{\prime 2} g_{\mathrm{OD}}\left(r^{\prime}\right) d r^{\prime}$ which directly depends on

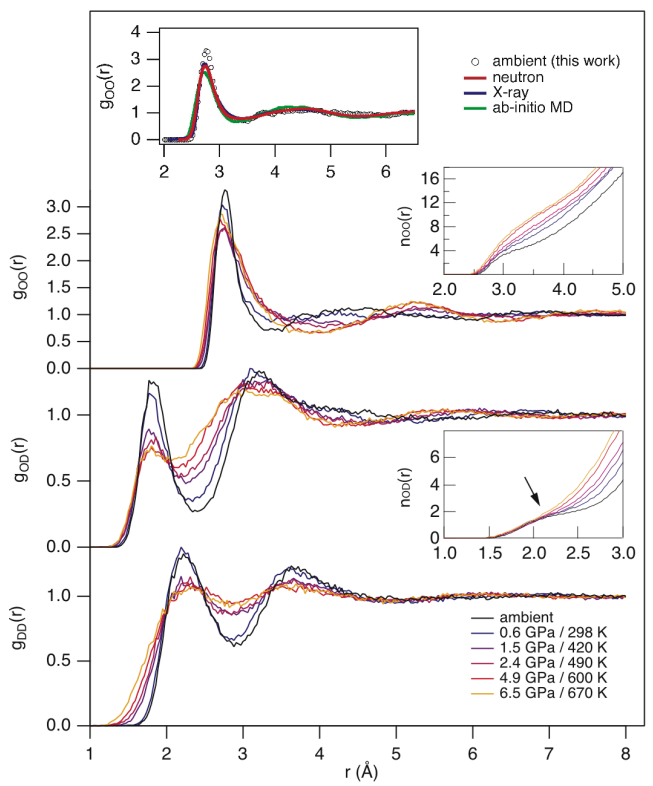

FIG. 2 (color online). Intermolecular partial radial distribution functions $g_{\alpha \beta}(r)$ as determined by EPSR reproducing the measured $S(Q)$ of $\mathrm{D}_{2} \mathrm{O}$ (Fig. 1). Upper inset: comparison of $g_{\mathrm{OO}}$ with earlier neutron [12], x-ray [19], and $a b$ initio MD [20] studies at ambient pressure. Right insets: coordination numbers $n_{\mathrm{OO}}(r)$ and $n_{\mathrm{OD}}(r)$; the arrow marks the distance up to which $n_{\mathrm{OD}}(r)$ shows no density dependence. 


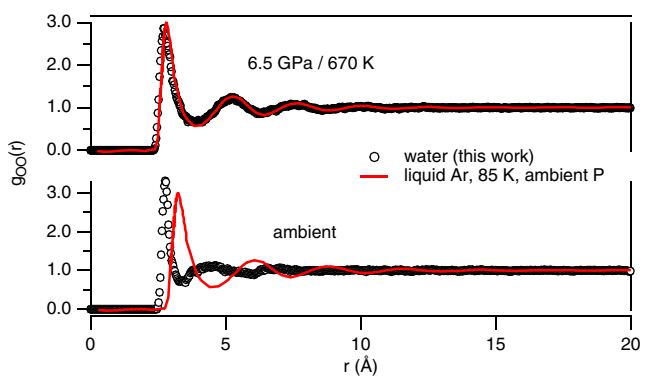

FIG. 3 (color online). Parameter-free comparisons between $g_{\mathrm{OO}}(r)$ of liquid water (open circles, from Fig. 2) and $g(r)$ of liquid argon (solid line). Upper curves: between $\mathrm{D}_{2} \mathrm{O}$ at 6.5 GPa/670 K $\left(\rho_{\mathrm{D}_{2} \mathrm{O}}=0.0510\right.$ atom $\left./ \AA^{3}\right)$ and Ar. Lower curves: between $\mathrm{D}_{2} \mathrm{O}$ at ambient conditions $\left(\rho_{\mathrm{D}_{2} \mathrm{O}}=\right.$ 0.0332 atom $/ \AA^{3}$ ) and Ar. The $g(r)$ of $\mathrm{Ar}$ was measured at $85 \mathrm{~K}$ (ambient pressure, $\rho_{\mathrm{Ar}}=0.0213$ atom $/ \AA^{3}$ ) [22] and scaled to equal mean number density $\left[r \rightarrow r \cdot\left(\rho_{\mathrm{Ar}} / \rho_{\mathrm{D}_{2} \mathrm{O}}\right)^{1 / 3}\right]$.

$\rho$, and as stated above, $n_{\mathrm{OD}}(r)$ remains unchanged up to $r \sim 2.2 \AA$.

In order to corroborate and quantify the conclusions emerging from Fig. 2, we now analyze the radial and angular distribution functions in terms of distance ranked groups of neighbors with respect to a given central molecule $[8,23]$. In the left of Fig. 4, we show distance distributions of four given groups. In order to reveal distance changes in excess to what is expected from the change of density, we plot the distance distributions of Fig. 4 versus distances that are scaled to ambient density $\rho_{0}$ [i.e., $r \rightarrow$ $r\left(\rho / \rho_{0}\right)^{1 / 3}$. Hence, for a group with distances decreasing in proportion to the density increase, we would expect no shift of the distribution, whereas rigid (soft) groups of neighbors can be identified by shifts to the right (left). In the right of Fig. 4, we show angular distributions with respect to the covalent $\mathrm{O}-\mathrm{H}$ bond of the central molecule (see right inset of Fig. 4). As expected, the group of first neighbors (1-4) apparently forms the tetrahedrally coordinated first shell with two characteristic peaks in the angular distribution and which becomes slightly angularly distorted at elevated $P / T$ [24]. This first shell of neighbors behaves rigidly (shift to the right of the rescaled distributions) with an almost density-independent absolute distance from the central molecule (seen also in the upper left inset of Fig. 4). The radial distribution of the second group (5-7) reveals a difference between ambient and elevated $P / T$ which may be associated with the gradual transformation towards HDW, while thereafter it approximately scales with density and becomes radially more localized. The angular distribution of this group as well as those of the last two groups are centered at $\sim 60^{\circ}$, which is known to occur in configurations of random-packed spheres [25]. However, in the distance distributions pronounced differences between the third and the fourth groups are found. Distances of the latter group (12-16)

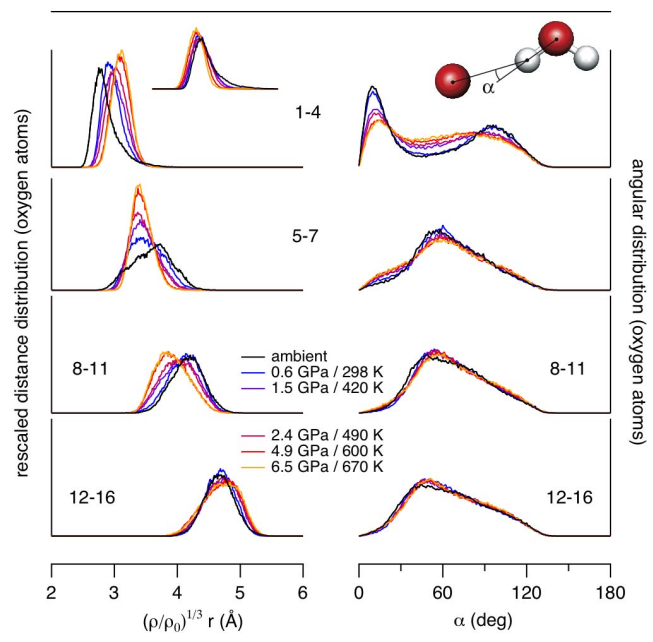

FIG. 4 (color online). Distance and angular distributions (see right inset for definition) of groups of 1 st to 4 th, 5 th to 7 th, 8 th to 11 th, and 12 th to 16 th oxygen neighbors, respectively. Note that distances are rescaled to ambient density (see text), except for the upper left inset depicting nominally scaled distributions of the 1st group.

decrease in proportion to the density increase (no shift of the rescaled distributions), which is expected in the limit of large distances. On the other hand, the distances of the third group (8-11) apparently compensate the relative incompressibility of the first shell (shift to the left of the rescaled distributions). The above ranked neighbor analysis thus gives clear evidence that the presence of $\mathrm{H}$ bonds still affects the local structure in water even at the highest $P / T$ and at least within a local distance range up to $\sim 5 \AA$ including next nearest neighbors. This finding supports the initial conclusion derived from the density-independent first plateau in $n_{\mathrm{OD}}(r)$ as mentioned above (Fig. 2) and the fact that we find the number of $\mathrm{H}$ bonds per molecule to decrease only by $\sim 10 \%$ up to the highest $P / T$ [26], in agreement with earlier $a b$ initio MD calculations at similar densities [15].

On the other hand, the apparent flexibility of the yet intact $\mathrm{H}$ bonds still allows the molecules of dense water to adopt a local structure commonly found in simple liquids, as shown by the almost perfect, parameter-free correspondence between the $g_{\mathrm{OO}}(r)$ of $\mathrm{D}_{2} \mathrm{O}$ at high $P / T$ and the $g(r)$ of liquid argon [22] shown in Fig. 3. To better understand this correspondence, we have carried out classical MD simulations using the TIP4P and the Lennard-Jones (LJ) potential at the same number-density and temperature [27]. Surprisingly, we find that the LJ MD simulation may reasonably well reproduce the structure of high $P / T$ water in both angular and radial distributions. This is supported by a so-called pair analysis of the oxygen atoms. This technique, described in detail in [28], allows characterizing the local structure of a disordered system by assigning specific indices to the geometric relation between two 


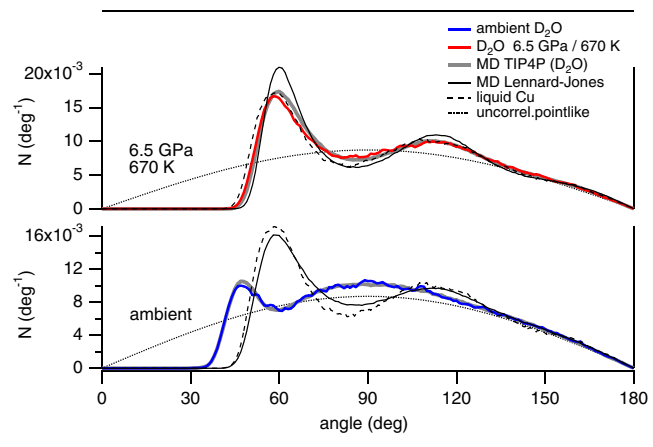

FIG. 5 (color online). Angular distributions within the 8 nearest oxygen neighbors compared with liquid copper [25] and MD simulations of water using the TIP4P and LJ potential. The dotted lines denote the distribution $\propto$ sin of uncorrelated pointlike particles.

given atoms. It thus enables us to compare quantitatively the experimental water with the LJ MD simulation: at high $P / T$ similar index values are found $\left(\chi^{2}=0.001\right)$, contrary to the case at ambient conditions $\left(\chi^{2}=0.035\right)$ [29]. All pair-analysis indices from the experimental box at high $P / T$ are similar to indices found in earlier studies on simple liquids [28]. Additionally, we may compare our experimentally determined angular distributions of the eight nearest oxygen neighbors with those of our TIP4P and LJ MD simulations, and with that of liquid copper determined by $\mathrm{x}$-ray absorption spectroscopy and calculated to a corresponding radial cutoff value [25]. Figure 5 illustrates that at high $P / T$ the angular distribution in $\mathrm{D}_{2} \mathrm{O}$ indeed matches closely those found for a perfect LJ liquid and liquid copper.

In summary, we conclude that with increasing density water approaches a local structure common to a simple liquid. However, the $\mathrm{H}$ bonds are found to remain intact; i.e., they influence the short range structure up to at least a radius of $5 \AA$ to a central molecule. The latter fact is reflected in the tetrahedral first-neighbor coordination, which is slightly deformed to accommodate the structure of a simple liquid. A similar structural evolution with increasing density was found in very high-density amorphous ice (VHDA), which approaches a random-closedpacked structure with yet intact $\mathrm{H}$ bonds [23]. Liquid water at high densities should thus be rather structurally related to VHDA than to ice VII.

This work was funded by the Swiss National Science Foundation (T.S.), a Marie Curie Fellowship of the EU (T.S.), and a research grant from EPSRC (J.S.L. and R. J.N.), and is supported by CCLRC through access to beam time and other resources. We are indebted to A. K. Soper for many stimulating discussions and valuable help and advice in the use of EPSR. We thank F. Datchi for the implementation of the Saul-Wagner equation of state and A. Trapananti for providing us data on liquid copper.

*Electronic address: thierry.straessle@psi.ch Present address: Laboratory for Neutron Scattering, ETH Zürich \& PSI, 5232 Villigen PSI, Switzerland.

[1] P. G. Debenedetti and H.E. Stanley, Phys. Today 56, No. 6, 40 (2003).

[2] P. H. Poole et al., Nature (London) 360, 324 (1992).

[3] R. J. Speedy, J. Chem. Phys. 86, 982 (1982).

[4] S. Sastry et al., Phys. Rev. E 53, 6144 (1996).

[5] H. Tanaka, J. Chem. Phys. 112, 799 (2000).

[6] A. K. Soper and M. A. Ricci, Phys. Rev. Lett. 84, 2881 (2000).

[7] T. Kawamoto, S. Ochiai, and H. Kagi, J. Chem. Phys. 120, 5867 (2004).

[8] A. M. Saitta and F. Datchi, Phys. Rev. E 67, 020201(R) (2003).

[9] A. Y. Wu, E. Whalley, and G. Dolling, Mol. Phys. 47, 603 (1982).

[10] A. V. Okhulkov, Y.N. Demianets, and Y.E. Gorbaty, J. Chem. Phys. 100, 1578 (1994).

[11] M.-C. Bellissent-Funel and L. Bosio, J. Chem. Phys. 102, 3727 (1995).

[12] A. K. Soper, Chem. Phys. 258, 121 (2000).

[13] M. Krisch et al., Phys. Rev. Lett. 89, 125502 (2002).

[14] P. Wernet et al., Science 304, 995 (2004).

[15] E. Schwegler, G. Galli, and F. Gygi, Phys. Rev. Lett. 84, 2429 (2000).

[16] Y. Le Godec et al., High Press. Res. 24, 205 (2004).

[17] S. Klotz et al., Phys. Rev. Lett. 89, 285502 (2002).

[18] A. Saul and W. Wagner, J. Phys. Chem. Ref. Data 18, 1537 (1989).

[19] G. Hura et al., J. Chem. Phys. 113, 9140 (2000).

[20] B. Hetényi et al., J. Chem. Phys. 120, 8632 (2004).

[21] B. Chen et al., Phys. Rev. Lett. 91, 215503 (2003).

[22] J. L. Yarnell, M. J. Katz, R. G. Wenzel, and S. H. Koenig, Phys. Rev. A 7, 2130 (1973).

[23] A. M. Saitta et al., J. Phys. Chem. 121, 8430 (2004).

[24] The angular distributions in Fig. 4 are not normalized to the distribution $\sin (\alpha)$ of pointlike particles. Normalization yields two peaks for the the first group at $0^{\circ}$ and $109.5^{\circ}$.

[25] A. Di Cicco et al., Phys. Rev. Lett. 91, 135505 (2003).

[26] We assigned two molecules as being $\mathrm{H}$ bonded if the $\mathrm{O}-\mathrm{O}$ distance was $\leq 3.5 \AA$ and the angle $\mathrm{O} \cdot \mathrm{H}-\mathrm{O} \geq 140^{\circ}$.

[27] TIP4P potential, 512 water molecules, NPT ensemble; LJ potential derived from TIP4P potential without Coulomb interactions.

[28] J. D. Honeycutt and H. C. Andersen, J. Phys. Chem. 91, 4950 (1987).

[29] $\chi^{2}$ denotes the sum of squared differences over the normalized [29] indices 1421, 1422, 1441, 1541, 1551, 1661, $2101,2211,2331,2441$ within the nearest 12 oxygen neighbors. 